\title{
ReGale: Some memorable results
}

\section{Joel Sobel}

Department of Economics, University of California, San Diego, La Jolla, CA 92093, USA

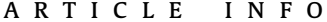

\section{Article history:}

Received 24 April 2009

Available online 5 May 2009
A B S T R A C T

This article reviews, with admiration and awe, the scientific contributions of David Gale.

(C) 2009 Elsevier Inc. All rights reserved.

\section{JEL classification:}

C61

C62

C65

C72

C78

\section{Contents}

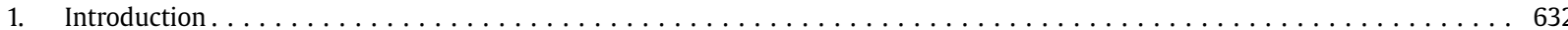

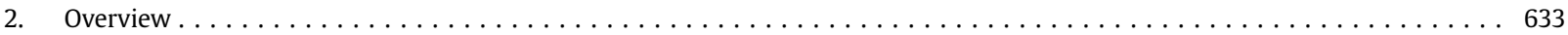

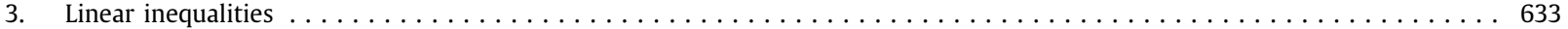

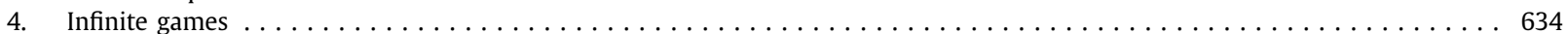

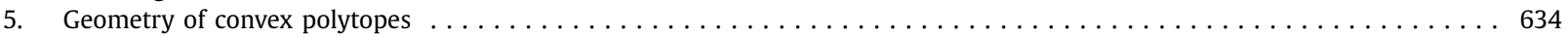

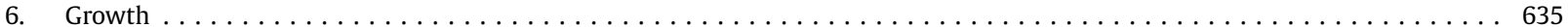

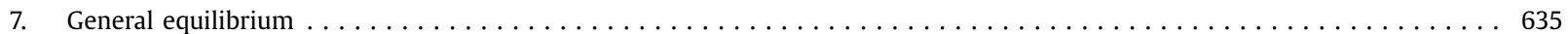

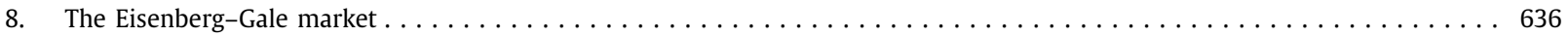

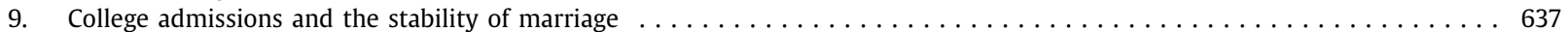

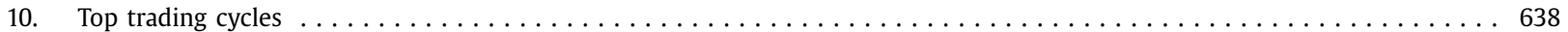

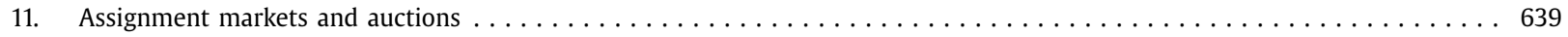

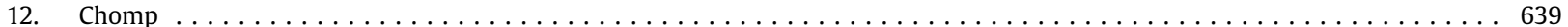

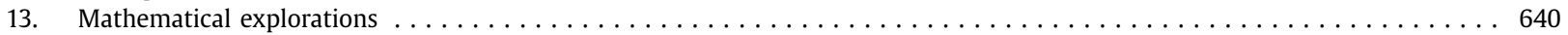

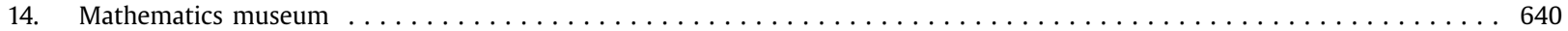

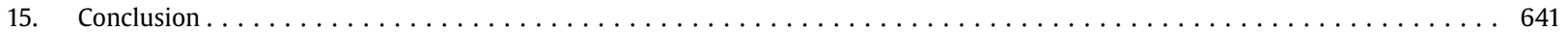

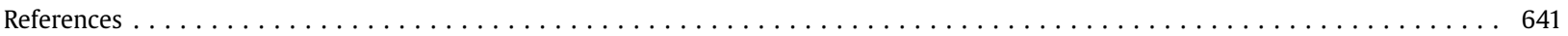

\section{Introduction}

David Gale published nearly 100 articles. Most are short. Several are masterpieces. One can add to these papers, but one cannot improve them. Gale had a genius for finding problems that were simple to state. He found elegant and elementary ways to solve these problems.

\footnotetext{
I I thank Antonio Cabrales, Jerome Coonen, Daryl Cooper, Gabrielle Demange, Harold Kuhn, Philip Neary, Bernhard von Stengel, and Vijay Vazirani for helpful comments and NSF for financial support.

E-mail address: jsobel@ucsd.edu.
} 
I cannot surpass Gale's exposition. This article tries only to display the amazing range and beauty of his contributions. One way to measure the importance and breadth of David Gale's research is to list some of the discoveries that carry his name. Gale and Stewart games are the simplest infinite zero-sum games. They are of historic importance to game theory and led to important developments in set theory. Gale transforms and Gale diagrams are representations of convex polytopes that have far-reaching implications in algebraic and projective geometry. The von Neumann-Gale model is a benchmark linear dynamic equilibrium model. The Gale-Nikaidô-Debreu lemma is the critical mathematical result needed for the proof of existence of competitive equilibrium. Eisenberg-Gale markets, a topic of recent interest among theoretical computer scientists, are a family of markets in which it is possible to find computationally efficient algorithms to compute equilibrium. The Gale-Shapley Marriage Model and associated Gale-Shapley Algorithm have far-reaching economic applications. The simple "Game of Gale" is entertaining as a diversion and a source of computationally difficult problems.

After a brief general overview of Gale's life, this article proceeds to sketch his contributions in several different areas.

\section{Overview}

David Gale (December 13, 1921-March 7, 2008) received an undergraduate degree from Swarthmore and a masters degree from the University of Michigan before earning a Ph.D. in Mathematics at Princeton. He taught at Brown University from 1950 through 1965 and then joined the faculty at the University of California, Berkeley. His principal appointment was in the Mathematics Department, but he maintained affiliations with the departments of Economics and Industrial Engineering and Operations Research.

Gale won wide recognition for his research. His awards included a Fulbright Research Fellowship, two Guggenheim Fellowships, elections to the American Academy of Arts and Science and the National Academy of Science, the Lester Ford Prize (for outstanding mathematical exposition), the John von Neumann Theory Prize (for fundamental contributions to operations research), and the Pirelli Internetional Award (for the Internet Mathematics Museum "MathSite").

Mukul Majumdar (1992) edited the volume Equilibrium and Dynamics: Essays in Honour of David Gale. The Int. J. Game Theory, Volume 36, Numbers 3-4, March, 2008 contains a collection of papers dedicated to David Gale on the occasion of his 85th birthday. This volume is edited by Marilda Sotomayor, who had also organized a scientific celebration in David's honor during the 18th Summer Festival on Game Theory in Stony Brook, July 12/13, 2007.

Gale lived in Berkeley, California and Paris, France with his partner Sandra Gilbert, renowned feminist literary scholar and poet. Her 2000 book of poetry Kissing the Bread included a section of poems she wrote for Gale called "When she was kissed by the mathematician." He had three daughters and two grandsons. Julie Gale, his former wife and the mother of his daughters, died in February 2008. ${ }^{1}$

\section{Linear inequalities}

When David Gale arrived at Princeton he had an opportunity to work with Albert Tucker and Harold Kuhn. ${ }^{2}$ This group made fundamental mathematical discoveries that are now standard background knowledge to economists. Together Gale, Kuhn, and Tucker won the 1980 von Neumann prize for work they began in the late 1940s. Their citation stated that they "played a seminal role in laying the foundations of game theory, linear and non-linear programming - work that continues to be of fundamental importance to modern operations research and management science."

Gale et al. (1951) contained the first complete proof of the duality theorem of linear programming and used the theorem to prove the minimax theorem of zero-sum, two-person game theory. This is the first of several early papers of Gale that use convex analysis rather than fixed-point arguments to prove existence theorems.

It is easy to describe the essence of the Gale, Kuhn, and Tucker proof of the minimax theorem of two-player zero-sum game theory. Let $U$ be an $m \times n$ matrix of payoffs to the row player. Let $e$ be an $m$ - or $n$-vector of ones. Consider the following pair of linear programming problems with variables $p \in \mathbb{R}^{m}, q \in \mathbb{R}^{n}$, and $v, w \in \mathbb{R}$ :

$$
\begin{aligned}
& \max -w \text { subject to } U q-e w \leqslant 0,-e^{\top} q=-1, q \geqslant 0, \\
& \min -v \quad \text { subject to } p^{\top} U-v e^{\top} \geqslant 0,-p^{\top} e=-1, p \geqslant 0 .
\end{aligned}
$$

Both problems are feasible. A solution $\left(q^{*}, w^{*}\right)$ to (1) gives a mixed strategy $q^{*}$ of the column player where she pays no more than $w^{*}$ in each row played by the row player, and a solution $\left(p^{*}, v^{*}\right)$ to (2) gives a mixed strategy $p^{*}$ of the row player where he gets at least $v^{*}$ in each column played by the column player. Problem (2) is the dual of (1). The duality theorem of linear programming implies that both problems have solutions with equal optimal values $-w^{*}=-v^{*}$, which gives the minimax theorem with $v^{*}$ as the value of the game.

The Theory of Linear Economic Models (Gale, 1960) remains a standard reference. The book contains central results on the theory of linear inequalities, including Gale et al. (1951) and Gale (1956a). It discusses Dantzig's simplex algorithm and

\footnotetext{
1 David counted his students as family members. We were sitting together in a Berkeley cafe in the early 1980s when his daughter Katharine entered. David introduced us by saying: "Joel, this is my daughter, Katharine. Katharine, this is my son, Joel." The Mathematics Genealogy Project lists 14 students who earned mathematics Ph.D.s under Gale's supervision. This list does not include students who earned Ph.D.s in other disciplines.

2 Tucker became Gale's dissertation supervisor. Gale and Kuhn began graduate studies at Princeton in the same year.
} 
gives an economic interpretation to canonical problems. The book also contains a concise and elementary introduction to the theory of linear inequalities (including a proof of the separating hyperplane for convex polytopes), a chapter containing essential results on non-negative matrices, and a clean treatment of dynamic linear models of growth. The book presents mathematics rigorously, but with an emphasis on problems in mathematical economics.

\section{Infinite games}

All two-player finite zero-sum games have a value. Gale and Stewart (1953) introduce a class of infinite zero-sum games and demonstrate that the minimax theorem need not hold.

Imagine a two-player game of perfect information in which the players, Alice and Bob, take turns announcing binary numbers. The game has a countable (but possibly infinite) number of rounds. The outcome of the game is a sequence $\left\{a_{0}, a_{1}, \ldots\right\}$ where $a_{i}$ is the $i$ th number announced (and Alice selects $a_{2 n}$ while Bob selects $a_{2 n+1}$ ). The outcome of the game can be viewed as an element of the unit interval. Each subset $A \subset[0,1]$ determines a two-player zero-sum game of perfect information. Alice wins if the outcome is in $A$. Bob wins otherwise. Call this game $\Gamma(A)$

Because there are no draws, the game must end in a win for Alice or a win for Bob. A game is determined if a player has a winning strategy. All finite games are determined. Gale and Stewart show that if $A$ is closed, then $\Gamma(A)$ is determined. The result has a straightforward intuition. If $A$ is closed, then any win for Bob will be reached after a finite number of moves. If Alice cannot find a first move that prevents Bob from winning, then Bob must be able to win no matter what Alice does. Otherwise Alice will always have a move that prevents Bob from finding a winning continuation. This observation leads immediately (by exchanging the roles of Alice and Bob) to the conclusion that $\Gamma(A)$ is determined when $A$ is open.

The natural question is to characterize the set of $A$ for which $\Gamma(A)$ is determined. Gale and Stewart use the Axiom of Choice to demonstrate the existence of a set $A$ for which $\Gamma(A)$ is not determined. That is, it is possible to construct a set A such that given any strategy of Alice, Bob has a winning strategy and given any strategy of Bob, Alice has a winning strategy. Hence the fundamental theory of two-person, zero-sum game theory does not hold for infinite games.

Gale and Stewart's result lead to research in two directions that have led to important developments in set theory. One direction identifies the set of games that are determined. Martin (1975) proved that if $A$ is a Borel subset of [0,1], then $\Gamma(A)$ is determined. ${ }^{3}$ Gale and Stewart's problem has lasting importance in set theory because the proof of the determinacy of Borel sets uses all of the axioms of set theory (Friedman, 1971).

The other direction investigates axiomatic foundations of set theory that replace the Axiom of Choice by an axiom that guarantees that $\Gamma(A)$ is determined for all sets $A$ (Mycielski, 1964).

\section{Geometry of convex polytopes}

Gale made lasting contributions to the study of convex polyhedra. While this work is not widely known to economists and has had a limited impact on game theory, the central issues arise from the study of combinatorial optimization problems and the proof techniques are methods from convex analysis.

Gale (1956b) identifies counterintuitive properties of the geometry of high-dimensional polytopes. A convex polytope $P$ is the convex hull of a finite set of points in some Euclidean space. The extreme points of $P$ are its vertices. The segment joining two distinct vertices of $P$ is an edge of $P$ if it is the intersection of $P$ and a supporting hyperplane, in which case these vertices are neighbors. More generally a subset $R=\left\{v_{1}, \ldots, v_{m}\right\}$ of vertices of a polyhedron $P$ are neighbors if the convex hull of $R$ spans a face of $P$. A polyhedron is $m$-neighborly if every subset of $m$ vertices is a set of neighbors.

In three-dimensional space, the only polyhedra that are 2-neighborly have at most four vertices. This observation suggests the conjecture that 2-neighborly polyhedra in higher dimensions have a limited number of vertices. A striking example due to Harold Kuhn (1955) cast doubt on the conjecture. Kuhn discovered that the Traveling Salesman Polytope ${ }^{4}$ for the asymmetric 5-city problem is 2-neighborly. This led Kuhn to ask: "What is the largest number of extreme points for a neighborly polytope in $n$ dimensions?" Gale provided the answer. He proved that for any $n>0$ there exists a convex polyhedron in $\mathbb{R}^{2 m}$ with $n$ vertices such that every $m$ of which are neighbors. In particular, the possibilities in dimension four (where, for all $n$, there is a convex polyhedron with $n$ vertices in which all pairs of vertices are neighbors) are much richer than in dimension three.

Gale (1963a) uses a family of cyclic polytopes to exhibit examples of neighborly polyhedra. Let $M=\left\{\theta(t) \in \mathbb{R}^{2 k}: \theta(t)=\right.$ $\left.\left(t, t^{2}, \ldots, t^{2 k}\right)\right\}$ be the moment curve. A cyclic polytope in $\mathbb{R}^{2 k}$ is the convex hull of $n>2 k$ points on $M$. Gale shows that for any distinct $t_{1}, \ldots, t_{n}$ the convex hull of $\left\{\theta\left(t_{1}\right), \ldots, \theta\left(t_{n}\right)\right\}$ is $m$-neighborly. He also proves the "Gale evenness condition": Given a cyclic polyhedron $P$, the set $R$ containing $2 m$ points from $P$ are neighbors if and only if every two vertices of $P$ that are not in $R$ are separated by an even number of points of $R .^{5}$

\footnotetext{
3 Wolfe (1955) proved determinacy for the second level of Borel games (for which $A$ is a countable union of closed sets).

4 The traveling salesman polytope is the convex hull of the incidence vectors of all complete directed tours. When there are 5 cities, there are 5 ! $=24$ tours. The incidence vectors can be viewed to lie in an 11-dimensional subset of $\mathbb{R}^{20}\left(\left\{\left(x_{i j}\right), 5 \geqslant i, j \geqslant 1, i \neq j: x_{i j} \geqslant 0, \sum_{j \neq i} x_{i j}=1\right.\right.$ for all $i$, and $\sum_{i \neq j} x_{i j}=1$ for all $\left.\left.j\right\}\right)$.

5 Gale (1956b) and Motzkin (1957) (who independently reported similar results) were not aware of much earlier work by Carathéodory (1907, 1911) that described cyclic polytopes and their properties.
} 
This result is useful for algorithmic game theory. Savani and von Stengel (2006) use the Gale evenness condition to construct examples in which the Lemke and Howson (1964) algorithm for solving bi-matrix games is exponential.

Gale (1956b) contains the central ideas of what are now known as the Gale transform and the Gale diagram. If $P$ is a polytope in $\mathbb{R}^{d}$ with $n$ vertices, then a Gale diagram of $P$ is a set of $n$ points in $\mathbb{R}^{n-d-1}$ that are in $1-1$ correspondence with the vertices of $P$. When $P$ has relatively few vertices $(n \leqslant 2 d)$ the diagram is a subset of a lower-dimensional space than $P$ and, in particular, when $n=d+3$, the Gale diagram is a subset of $\mathbb{R}^{2}$. Consequently, the transform makes it possible to visualize high-dimensional polytopes with few vertices. ${ }^{6}$

\section{Growth}

The von Neumann-Gale model of growth (developed in Gale (1956a) and Gale (1960, pp. 310-315)) generalizes and simplifies the von Neumann (1937) $)^{7}$ model of an expanding economy. Gale substituted von Neumann's requirement that each production process involves each good in the economy (as either input or output), with a weaker, more plausible condition. He also used convex analysis (rather than fixed-point arguments) to provide short, elementary proofs of the main results.

Gale (1967) provides the definitive treatment of the multi-good Ramsey problem. Given an abstract set of economic states $S$, and a subset $\mathcal{T} \subset S \times S$ called the technology, a program is a sequence $\left\{s_{t}\right\}, t=0,1, \ldots, T$ such that $\left(s_{t}, s_{t+1}\right) \in \mathcal{T}$ for all $t=0, \ldots, T-1$. Assume that the program generates a sequence of utilities, $u_{t}$. The problem is to maximize $\sum_{t=0}^{T} u_{t}$ subject to $s_{0} \in S_{0}$, where $S_{0}$ is a given set of initial states. The von Neumann linear production technology is easily placed into this framework.

The existence problem is straightforward as stated above with finite $T$. Difficulties arise when the horizon is infinite because the objective function may diverge. Gale works with the overtaking criterion: An infinite program $\left\{s_{t}\right\}, t=0, \ldots$, is said to overtake a program $\left\{s_{t}^{\prime}\right\}$ if there exists a $T$ such that $\sum_{\tau=1}^{T}\left(u_{\tau}-u_{\tau}^{\prime}\right)>0$ for all $t>T$. A weaker comparison is the criterion of catching up: $\left\{s_{t}\right\}, t=0, \ldots$, is said to catch up to a program $\left\{s_{t}^{\prime}\right\}$ if $\liminf _{T \rightarrow \infty} \sum_{\tau=1}^{T}\left(u_{\tau}-u_{\tau}^{\prime}\right) \geqslant 0$. The problem is to find a program $\left\{s_{t}\right\}$ that is optimal (strongly optimal) from $S_{0}$ in the sense that $\left\{s_{t}\right\}$ catches up to (overtakes) any other program $\left\{s_{t}^{\prime}\right\}$ with $s_{0}^{\prime} \in S_{0}$. Optimal programs need not exist and limits of finite-horizon optimal programs need not be optimal. Gale presents conditions under which optimal programs exist and a characterization in terms of "turnpike" properties. Roughly speaking, one can construct an optimal program with two phases: a bounded initial transition phase in which the state is built up to (approximate) a sustainable optimal steady-state followed by a program that approximates the best steady-state consumption.

Gale (1973) is an important extension of Samuelson's (1958) overlapping-generations model. When agents live for two periods, these models typically have multiple equilibria: balanced growth paths in which agents consume their endowment in each period; and optimal golden-rule paths where the interest rate is equal to the population growth rate and where, in general, there are intergenerational transfers. Gale formalizes these ideas, extends them to economies in which agents live for $n$ periods, and studies the stability properties of equilibrium. This paper is among the first to point out the indeterminacy of equilibrium in dynamic models, an issue that has received a lot of attention by macroeconomists. In particular, the paper shows how indeterminacy depends on the life span of agents and the existence of aggregate debt.

\section{General equilibrium}

Gale make several important contributions to the foundations of general-equilibrium theory.

Gale (1955) contains a result known as the Gale-Debreu-Nikaidô lemma (Debreu, 1956, and Nikaidô, 1956) that provides the essential mathematical result needed to prove existence of market equilibrium. Gale shows that if $\phi: \Delta \rightarrow \mathbb{R}^{m}$ is a nonempty, convex, compact-valued upper hemi-continuous correspondence from the $n$-dimensional unit simplex $\Delta$ such that $p \cdot z \leqslant 0$ for all $z \in \phi(p)$, then there exists $\left(p^{*}, z^{*}\right) \in \Delta \times \mathbb{R}^{m}$ such that $z^{*} \leqslant 0$. In the application to general equilibrium, $\phi(\cdot)$ is the excess-demand correspondence and $p \cdot z \leqslant 0$ is Walras's Rule. The price vector $p^{*}$ leads to non-negative excess demand. Gale's proof uses the Knaster, Kuratowski, and Marzurkiewicz theorem while Debreu (1956) and Nikaidô (1956) use the Kakutani fixed-point theorem.

Gale and Nikaidô (1965) proves a theorem on the global univalence of differentiable mappings on $\mathbb{R}^{n}$. The main result is: Let $S$ be a closed rectangular subset of $\mathbb{R}^{n}$ and $F: S \rightarrow \mathbb{R}^{n}$ be a differentiable on $S$ with Jacobian matrix $J$. If $J(x)$ is a $P$-matrix ${ }^{8}$ for all $x \in S$, then $F$ is univalent. When translated into a general-equilibrium context, the theorem gives sufficient conditions for equilibrium prices to be unique (see, for example, Arrow and Hahn, 1971, Chapter 9).

Gale (1963b) provides an early, robust example of global instability of the tâtonnement process in general equilibrium.

Gale and Mas-Colell (1975) provides an existence theorem in an economy without ordered preferences.

\footnotetext{
${ }^{6}$ Grünbaum (2003) contains a description of the transform and its implications for the classification of polytopes. In the 1966 edition of his book, Grünbaum (2003, p. 85) writes "the reader will find it well worth his while to become familiar with the concepts of Gale-transforms and Gale-diagrams. ... It is very likely that the method will yield many additional results." The additional notes section, added for the second edition, confirms the importance of the methods and lists references (Grünbaum, 2003, p. 96a). Eisenbud and Popescu (2000) describes generalizations and applications to algebraic geometry.

7 von Neumann (1945-1946) is the English translation.

8 A square matrix is a $P$-matrix if all of its principal submatrices have positive determinant.
} 


\section{The Eisenberg-Gale market}

Eisenberg and Gale (1959) assume that there are $m$ bettors and $n$ horses. Bettor $B_{i}$ believes that the probability that Horse $H_{j}$ will win the race is $p_{i j}$, where $p_{i j} \geqslant 0$ for all $i$ and $j$ and $\sum_{j=1}^{n} p_{i j}=1$ for all $i$. Bettor $B_{i}$ has a positive stake, $b_{i}$, to bet. Let $\pi_{j}$ represent the pari-mutuel odds for $H_{j}$. That is, if $B_{i}$ bets $b_{i j}$ on $H_{j}$, then he will receive $b_{i j} / \pi_{j}$ if $H_{j}$ wins the race and lose his bet otherwise. He will select a bet that maximizes his expected winnings. Consequently, $B_{i}$ will bet only on a horse $j$ that maximizes $p_{i j} / \pi_{j}$. Assume that each bettor spends all of his stake $\left(\sum_{j=1}^{n} b_{i j}=b_{i}\right)$ and for each horse there is a bettor who believes the horse has a chance to win (for each $j, p_{i j}>0$ for some $i$ ). For convenience, normalize the total stake to be one $\left(\sum_{i=1}^{n} b_{i}=1\right)$.

Eisenberg and Gale asked whether it was possible to find a probability distribution $\pi=\left(\pi_{1}, \ldots, \pi_{n}\right)$ such that

$$
\pi_{j}=\sum_{i=1}^{m} b_{i j}
$$

Eq. (3) is an equilibrium condition. It states that the betting odds for $H_{j}$ are equal to the fraction of money wagered on $H_{j}$ (remember that the total amount wagered is normalized to one). In this way, $\pi$ is a consensus probability distribution (where individual beliefs are weighted by relative wealth, $b_{i}$ ). Showing that (3) has a solution is a straightforward fixedpoint problem. Eisenberg and Gale's contribution was to characterize the unique consensus probability distribution as a solution to an optimization problem. They did this by introducing the function

$$
\phi(x)=\sum_{i=1}^{m} b_{i} \log \left(\sum_{j=1}^{n} p_{i j} x_{i j}\right)
$$

defined on the domain $X \subset \mathbb{R}^{m n}$,

$$
X=\left\{x=\left(x_{i j}\right) \in \mathbb{R}^{m n}: x_{i j} \geqslant 0 \text { and } \sum_{j=1}^{n} x_{i j}=1 \text { for all } i\right\} .
$$

The function $\phi(\cdot)$ has a unique maximum on $X$. Furthermore, it is not difficult to show that if $x^{*}$ solves:

$$
\max \phi(x) \text { subject to } x \in X \text {, }
$$

then

$$
\pi_{j}=\max _{i} \frac{b_{i} p_{i j}}{\sum_{j=1}^{n} p_{i j} x_{i j}^{*}} \quad \text { and } \quad b_{i j}=\pi_{j} x_{i j}^{*}
$$

constitute optimizing bets and odds that satisfy (3). The uniqueness argument is also elementary. ${ }^{9}$

This paper stimulated a literature on how to reach a consensus (for example, DeGroot, 1974) and has recently been influential in the work of theoretical computer science. Theoretical computer scientists have recently criticized solution concepts from mathematical economics because they are non-algorithmic. ${ }^{10}$ This concern has led to a research program designed to identify market environments in which equilibria can be computed or approximated using polynomial-time algorithms. Jain and Vazirani (2007) describe several linear environments in which polynomial-time algorithms for computing equilibria exist. These environments are related because in each of them equilibria can be characterized as a solution to an optimization problem. In fact, Jain and Vazirani define an Eisenberg-Gale-type convex program (maximizing (4) subject to $x \in X$ is a canonical example) and identify efficient algorithms for finding equilibria in markets in which equilibrium allocations solve an Eisenberg-Gale-type program. ${ }^{11}$

A related problem led to my only joint project with Gale. When I was a graduate student, David asked me the following question. Given an economy with a single homogeneous good, $m$ agents, and uncertainty about the total supply, was it possible to come up with a state contingent distribution that was Pareto efficient and fair in the sense that each agent's expected distribution was equal to a predetermined share. More precisely, an economy consisted of a probability distribution $p=\left(p_{1}, \ldots, p_{n}\right)$ defined over a finite set $S$ of $n$ states of the world; a vector $h=\left(h_{1}, \ldots, h_{n}\right)$, where $h_{j}>0$ is the total resource available in state $j$; shares $\theta=\left(\theta_{1}, \ldots, \theta_{m}\right) \geqslant 0$, such that $\sum_{i=1}^{m} \theta_{i}=1$, and utility functions $u_{i j}(\cdot)$ for each $i$, where

\footnotetext{
9 David presented the results of Eisenberg and Gale (1959) and Gale and Sobel (1982) at a Mathematics Department colloquium at Berkeley. The talk was advertised widely and because the title included the words "Pari-mutuel Betting" it attracted a few people who were not members of the Mathematics Department. One of these people was agitated when David noted that $\sum_{j=1}^{n} \pi_{j}=1$. "How can that be," he asked, "isn't $\pi=3.14 \ldots$ ?" David answered politely, but the confused horse-racing fan soon left the room.

10 Daskalakis et al. (2006) establishes that the related problem of computing Nash equilibria in four-player games is hard (more precisely, it is PPAD complete). Similar results exist for two- (Chen and Deng, 2006) and three-player (Daskalakis and Papadimitriou, 2005) games.

11 It is straightforward to transform the pari-mutuel betting model into a model of a linear exchange economy (see Gale, 1960, Chapter 8, Section 5). In this interpretation, the horses are commodities, the bettors are agents. Bettor $B_{i}$ has a linear utility function ( $p_{i j}$ is marginal utility of commodity $j$ ) and a budget of $b_{i}$. Under this interpretation, the optimization problem (5) characterizes equilibrium prices.
} 
$u_{i j}\left(x_{i j}\right)$ is Agent $i$ 's utility when he consumes $x_{i j}$ in state $j$. The problem then is to investigate the existence of a distribution scheme $x=\left(x_{i j}\right)$ that is feasible:

$$
\sum_{i=1}^{m} x_{i j}=h_{j} \text { for all } j \text { and } x \geqslant 0,
$$

proportional:

$$
\sum_{j=1}^{n} x_{i j}=\theta_{i} \sum_{j=1}^{n} p_{j} h_{j} \quad \text { for all } i,
$$

and efficient in the sense that there is no other feasible distribution scheme that increases the expected utility of all agents. When the utility functions are strictly increasing and strictly concave in consumption, it turns out that there is a unique fair distribution. David was not happy with my non-constructive proof and came up with a maximization problem analogous to the one that worked in the model of pari-mutuel betting. He discovered a constructive argument using the function

$$
\Phi(x)=\sum_{i=1}^{m} \sum_{j=1}^{n}\left(\int_{1}^{x_{i j}} \log u_{i j}^{\prime}(t) d t\right) p_{j} .
$$

The function $\Phi(\cdot)$ is defined on the set of feasible distribution schemes. Our joint paper (Gale and Sobel, 1982) shows that a distribution scheme is proportional and efficient if and only if it maximizes $\Phi$ over all distribution schemes. As in his paper with Eisenberg, Gale had come up with an unexpected optimization problem that characterized the solution to an economic problem.

\section{College admissions and the stability of marriage}

Gale's paper with Lloyd Shapley on the Stable Marriage Problem is his most cited, and probably most influential work. ${ }^{12}$ The short, deceptively simple paper is wonderful for several reasons. The motivation for the problem comes from the real world. The translation of the practical problem into mathematics captures many important considerations but remains extraordinarily simple. The solution to the problem is not obvious, but is easy to understand. The framework lends itself to modifications that lead to insight into more complicated practical problems. The underlying formal structure leads to mathematical results of interest to pure mathematics, game theory, and the study of algorithms.

Imagine two disjoint sets, $B=\left\{b_{1}, \ldots, b_{n}\right\}$ of $n$ boys and $G=\left\{g_{1}, \ldots, g_{n}\right\}$ of $n$ girls. Each girl has strict preferences over the elements of $B$. Each boy has strict preferences over the elements of $G$. Everyone wants to be matched with one member of the opposite sex. The problem is how to do it. In this simple setting, a matching can be thought of as a permutation $\pi$ of the first $n$ integers, with the interpretation that $b_{i}$ is matched with girl $g_{\pi(i)}$. Gale and Shapley show that it is possible to find a matching $\pi$ that is stable in that there is no unmatched pair $\left(b_{i^{*}}, g_{j^{*}}\right)$ such that $b_{i^{*}}$ prefers $g_{j^{*}}$ to $g_{\pi\left(i^{*}\right)}$ and $g_{j^{*}}$ prefers $b_{i^{*}}$ to $g_{\pi^{-1}\left(j^{*}\right)}$. In fact, they present the deferred-acceptance algorithm that converges to a stable match in a finite number of steps.

\section{Gale-Shapley Algorithm:}

Step 1. Each boy "proposes" to the favorite girl on his list.

Step 2. Each girl who receives at least one proposal, "dates" the boy she prefers among those who propose; she "rejects" the rest. Girls with no proposals do nothing.

Step 3. If no boy is rejected, stop. You have a stable matching between girls and their current dates. Otherwise, rejected boys cross the name of the girl who rejected them off their list and then propose to the favorite among those remaining. Boys who are "dating" repeat their proposal.

Step 4. Return to Step 2.

This algorithm is well defined, stops in a finite number of steps, and terminates in a stable matching. (Each boy proposes to - and was rejected by - all the girls he likes better than his final match. Every girl rejected him did so because she received an offer from someone she liked better.) What is more, the algorithm identifies a stable match with the property that all boys prefer their partner in this match to the partner that they would have received in any other stable match. In fact, the set of stable marriages is a lattice ${ }^{13}$ : Given two stable matchings $\pi$ and $\pi^{\prime}$ one can construct another stable matching by pairing each $b_{i}$ with his favorite of $\pi(i)$ and $\pi^{\prime}(i)$

\footnotetext{
12 The literature on stable matching is enormous. This section touches upon the direct contributions of David Gale. Broader surveys are available. Knuth (1976) (English translation: Knuth, 1997) is a research monograph describing aspects of the problem of interest to computer scientists. Gale (2001) and Roth (2008) are recent surveys. Roth and Sotomayor (1990) is an excellent reference on the theory of matching.

13 Gale (2001) and Roth and Sotomayor (1990) attribute this observation to Conway.
} 
Gale returned to the matching problem twenty years after the publication of the paper with Shapley. The paper of Gale and Sotomayor (1985) describes a general version of the marriage model in which the list of acceptable partners need not be complete (so that, for example, one girl may prefer to be unmatched than to be paired with some of the boys) and there may be different numbers of boys and girls. In this environment, it is clear that some players may be unmatched in a stable matching. Gale and Sotomayor prove the surprising property that if an agent is matched in one stable match, then he or she is matched in all stable matches.

Dubins and Freedman (1981) introduce the idea of strategic behavior in the stable matching problem. They ask whether an agent can gain by lying about their preferences in the deferred-acceptance algorithm. They show that when boys make proposals (and so that algorithm selects the stable matching that is optimal for the boys), boys cannot gain by changing the order in which they make proposals. Typically girls can gain by strategically rejecting the best proposal available in one round in order to obtain a still better proposal later. Another contribution of Gale and Sotomayor (1985) is a simple proof of this result. ${ }^{14}$

Starting in 1951, eleven years before the publication of the Gale-Shapley paper, the National Intern Matching Program used an essentially equivalent algorithm to match graduating medical students to hospital residency programs. ${ }^{15}$ There is now a long list of applications of the matching models. The theory has made it possible to design effective procedures in a variety of real-world settings. In turn, the applications have generated new questions and challenges for the theory. Gale and Shapley created one of the liveliest and most successful areas within game theory.

Marriage and assignment problems appear in different forms in another observation made by David Gale. An article by Gina Kolata (2007a) in The New York Times publicized Gale's skepticism about published surveys reporting that men have substantially more sexual partners than women. Gale pointed out that simple counting arguments make the surveys logically inconsistent. The newspaper article actually states and proves Gale's "High School Prom Theorem." Gale asks the reader to imagine that after a high school prom, each girl is asked to give the number of boys she danced with. These numbers are then added up giving a number $G$. The same information is then obtained from the boys, giving a number $B$. The theorem is: $B=G$. The proof is that both $B$ and $G$ must be equal to the number of couples that danced together (assuming there are no same-sex dancing pairs). $B=G$ is a necessary, but not sufficient, condition for the reported dance partners of the girls and boys to be consistent (in the sense that there exists a set of dance pairings such that the number of dances by each boy and girl is correct). For example, if one boy claimed to have danced with ten girls, then at least ten girls must claim to have had one partner. The main result in Gale (1957), which deduces a generalization of Hall's marriage theorem (Hall, 1935) from the max flow-min cut theorem, can be viewed as providing (non-trivial) necessary and sufficient conditions for the individual reports to be consistent. The original article about the High School Prom Theorem in The New York Times confused some readers (and led to a clarification published one week later, Kolata, 2007b). ${ }^{16}$

\section{Top trading cycles}

Shapley and Scarf (1974) credit David Gale with the idea of top trading cycles. Scarf and Shapley prove the core of a particular assignment game is non-empty. In their model there are $n$ traders and $n$ indivisible commodities. Agent $i$ originally owns commodity $i$. She obtains utility $a_{i j}>0$ if she receives $j$ (but she can only use one object). The core of this market consists of those assignments of people to objects with the property that there is no subset $S$ of traders who can redistribute their endowments in a way that makes all $i \in S$ strictly better off than in the given assignment. Scarf and Shapley demonstrate that the core of this game is not empty by showing that the game is balanced and applying Scarfs general existence theorem. ${ }^{17}$ Gale $(1974$, p. 30) provided an elementary and constructive way to generate a core allocation. For all $R \subset N$ let a top trading cycle for $R$ to be any non-empty $S$ subset of $R$ whose $S$ members can be ordered as $i_{1}, \ldots, i_{S}$ so that each individual $i_{k} \in S$ likes the $i_{k+1}$ th good at least as well as any other good in $R$. $^{18}$ Any non-empty $R \subset N$ has at least one top trading cycle. To see this, start with any trader in $R$ and construct a chain of most preferred goods. Because there is a finite set of traders, the chain must eventually repeat an element. Of course, if the trader selected first prefers her endowment to all others in $R$, the cycle will contain just one element. Consequently, it is possible to partition $N$ into $\left\{S_{1}, \ldots, S_{l}\right\}$ : Start with an arbitrary top-trading cycle $S_{1}$ for $N$ and continue inductively by letting $S_{r}$ be a top trading cycle of $N \backslash\left\{S_{1} \cup S_{2} \cup \cdots \cup S_{r-1}\right\}$.

The top trading cycle generates an allocation in a natural way. It is straightforward to show that this allocation is in the core. The idea of top trading cycles plays an important role in the construction of matching algorithms (see Abdulkadiroglu and Sönmez, 1999 and Roth et al., 2004).

\footnotetext{
14 The manipulability results are properties of underlying game and not the algorithm. In particular, Roth (1982) shows there is no non-manipulable mechanism that implements a stable matching.

15 See Roth and Sotomayor (1990, pp. 169-170) and Roth (2008, Appendix) for a discussion of the independent development of the matching algorithm.

16 Gale received many naive responses to the article and wrote to me that "I am spending a lot of time answering e-mail questions about sex."

17 In Shapley and Scarf (1974, Footnote 10, p. 35) there is a mysterious reference to the New Yorker (September 11, 1971, p. 91). This page of the New Yorker contains a description of the deferred acceptance algorithm credited to the Communications of the Association for Computing Machinery. The description ends with the sentence "All the women keep a dummy man 0 in suspense initially." To which an unnamed New Yorker writer added the comment "It sounds not unlike the old days."

18 By convention, $i_{s+1} \equiv i_{1}$.
} 


\section{Assignment markets and auctions}

Gale's work shows a sensitivity to computational issues. Knowing the connection between zero-sum, two-person game theory and the theory of linear programming combined with computational methods (like the simplex method) provides a tractable method for computing equilibria in two-player games. Demonstrating the equivalence between an equilibrium and the solution to a well behaved optimization problem is the reason that equilibria in Eisenberg-Gale markets can be found efficiently. His work on markets with indivisible goods is another example of a situation in which Gale adds just enough structure to a general model to obtain strong results. Existence and sensitivity results follow from constructive arguments.

Shapley and Shubik (1971) introduce the assignment model, a market equilibrium model with indivisible goods. Their model has the structure of an assignment game with the added feature that agents can exchange a divisible commodity, money. Demange and Gale (1985) look at a generalization of the basic assignment model in which Buyer $i$ receives utility $u_{i j}(x)$ if he receives item $j$ and the net transfer $x$. Demange and Gale characterize core allocations. ${ }^{19}$ This market inherits many properties from the college admissions problem. In particular, the set of equilibrium prices is a lattice. The lattice property guarantees that there is a "minimum-price" equilibrium. This equilibrium has the property that the price vector provides a weakly smaller price for each good than any other equilibrium price vector. Hence it is the equilibrium preferred by all buyers.

Demange and Gale study a strategic version of this game in which all agents submit demand functions and the outcome is the minimum-price competitive equilibrium. They show that this allocation is unique and not subject to manipulation by the buyers. Sellers, on the other hand, can manipulate prices (they are able to induce their most preferred equilibrium).

Alkan et al. (1991) use the assignment model to derive surprising fairness properties of equilibrium allocations. In an economy in which agents must allocate a finite number of indivisible objects and a fixed supply of (perfectly divisible) money, they show that when the supply of money is large enough, the set of efficient, envy-free allocations is non-empty. Because of the structure of the equilibrium set, these allocations satisfy strong, intuitive monotonicity properties (increasing the available money increases the welfare of all agents), that fail in more general models.

The paper Demange et al. (1986) applies the Shapley-Shubik framework to the study of multi-unit auctions. Consider an economy in which buyers have quasi-linear utilities and wish to buy at most one object, while each seller owns one object. An equilibrium is a price vector that equates supply and demand. A modified Vickery auction provides a mechanism that implements the minimum-price equilibrium. Demange, Gale, and Sotomayor describe two simple, dynamic procedures that implement this equilibrium.

The first algorithm assumes that valuations and prices are elements of a discrete set. It starts with prices equal to the sellers' reservation prices. Each buyer announces his utility-maximizing demands at these prices. If it is possible to meet these demands, then the reservation prices are equilibrium prices. If not, then there must be a set of objects for which there is excess demand. Next the seller raises by one unit the prices on all items in a minimal set of objects with excess demand. ${ }^{20}$ This process converges to equilibrium prices after a finite number of iterations. Demange, Gale, and Sotomayor show that these are minimal equilibrium prices.

The second algorithm (first studied by Crawford and Knoer, 1982) begins in the same way with the sellers setting prices equal to their reservation prices. The buyers now move according to an exogenously specified sequence. Each buyer may bid for any item. If he does so, then he commits himself to buying the item at the announced price and becomes the tentative owner of the item. At a general stage of the auction, some subset of items will be tentatively owned, and buyers will be partitioned into the set of tentative owners and the set of uncommitted buyers. The next uncommitted buyer in the sequence can do one of three things. (1) He can bid for an item that does not have a tentative owner (in which case he becomes the tentative owner at the current price). (2) He can bid for a tentatively owned item (in which case he becomes the tentative owner at the current price plus a fixed increment $\delta>0$ ). At this point the previous tentative owner is freed from his commitment to buy and becomes an uncommitted buyer. (3) He may drop out. The process continues. Plainly this process converges in a finite number of steps. Demange, Gale, and Sotomayor demonstrate that the limit approximates the minimum-price equilibrium (independent of the way the buyers are ordered). The approximation can be made arbitrarily good by reducing $\delta$.

While the results of Gale and his co-authors establish parallels between the theory of auctions and the theory of matching, the deeper relationships between the areas is still a subject of active research. Hatfield and Milgrom (2005) is an excellent place to look for results that synthesize the two literatures.

\section{Chomp}

There was a two-day conference in David Gale's honor organized by Marilda Sotomayor and held in Stony Brook, July 12/13, 2007. Gale's presentation, which appears as Gale (2009) in this issue, is a history of the board games invented by Gale and his contemporaries at Princeton. Gale's article provides an introduction to Bridg-It (or, as Martin Gardner called it, the "Game of Gale") and also John Nash's game of Hex. Gale (2009) does not describe another invention, Chomp.

\footnotetext{
19 They assume that there is a non-zero transfer between Buyer $i$ and the seller of good $j$ only if Buyer $i$ receives good $j$.

20 The existence of such a set follows from the theorem of Hall (1935). It is possible to find such a set using linear-programming methods.
} 
Chomp is a two-player game of perfect information. In the original version, introduced in Gale (1974) (see also Gale, 1998, Chapter 11) there is an $m \times n$ bar of chocolate sitting on the coordinate plane and the square $(0,0)$ is the lower left-hand corner. Players alternate moves, where a move consists of “chomping” by selecting a square with southwest corner ( $a, b$ ) that has not yet been eaten and eating all squares that are above and to the right. The last square (with corner $(0,0)$ ) is poisoned, so the player who selects that square loses. Since this is a finite game of perfect information, there must be a strategy that guarantees a win for either the first or second mover. The first mover must have a winning strategy: Consider removing the top-right square as the first move. Either this is already winning, or else the second player has a winning counter-move, which the first player could "steal" as his first move and thus leave the second player with a losing position.

When the chocolate bar is a square, the first mover must begin by removing the upper-right $(m-1, m-1)$ square, leaving an $L$-shaped portion. Subsequently, the first player simply mirrors the second player's move, always leaving the second player with an $L$ that has equal sides. When the chocolate bar is $2 \times n$, it is optimal for the first mover to remove only the upper right square and subsequently to leave player two with a choice in which one row is exactly one square longer than the other. These are the unique winning strategies.

Subsequent work has been done to find winning strategies and conditions under which winning strategies are unique. Higher-dimensional versions of the game or games in which the chocolate bar is not rectangular (so it is not clear which player has a winning strategy) have been studied.

\section{Mathematical explorations}

Gale and Shapley paper on "College Admissions and the Stability of Marriage" (1962, p. 15) concludes:

What, then, to raise the old question once more, is mathematics? The answer, it appears, is that any argument which is carried out with sufficient precision is mathematical, and the reason that your friends and ours cannot understand mathematics is not because they have no head for figures, but because they are unable to achieve the degree of concentration required to follow a moderately involved sequence of inferences. This observation will hardly be news to those engaged in the teaching of mathematics, but it may not be so readily accepted by people outside the profession. For them the foregoing may serve as a useful illustration.

Much of David Gale's academic research illustrates this definition of mathematics, but after he retired from his faculty position, Gale worked hard to make examples of beautiful mathematical arguments accessible to a broad audience.

Between 1991 and 1996 he wrote a column entitled Mathematical Explorations for The Mathematical Intelligencer. The columns, collected in a book titled Tracking the Automatic Ant, are in the tradition of Martin Gardner's long-running "Mathematical Games" column in Scientific American. ${ }^{21}$ These short essays contain novel problems in several areas of mathematics (geometry, number theory, and game theory). Some revisit earlier publications. All of the articles start with a tangible, elementary motivating problem. Many touch upon deep results. Anyone interested in getting an idea of Gale's life-long interest in puzzles and "recreational mathematics" should take a look a these essays.

\section{Mathematics museum}

In 2004, after a long gestation period, David Gale developed MathSite, a pedagogic website that uses interactive exhibits to illustrate important mathematical ideas. MathSite won the 2007 Pirelli Internetional Award for Science Communication in Mathematics.

MathSite (http://mathsite.math.berkeley.edu/main.html) currently consists of four "exhibits": Dissecting Triangles and Squares, Sorting Bricks and Sticks, Geometric Orbits, and the Stable Marriage Problem.

"Dissecting Triangles and Squares" illustrates the Hadwiger and Glur (1951) theorem that states that any two polygons with equal areas could be equidisected using only translations and halfturns. Visitors to the website can hear David Gale's voice introduce the exhibit. They can re-assemble polygons and, if they pay attention, will learn a constructive proof of the result. $^{22}$

"Sorting Bricks and Sticks" illustrates a variety of sorting algorithms. Visitors learn how to identify efficiently the heaviest (or longest) object when only binary comparisons are permitted. The exhibit also demonstrates more complicated sorting problems.

The exhibit about orbits lets the visitor simulate the behavior of a family of dynamical systems. Geometrically, the system begins with a convex polygon, a point inside the polygon, and an ordering of the sides of the polygon. The law of motion is to project the point to a side in the given order (repeating indefinitely). After the visitor sets a number of parameters (the number of sides in the polygon; the location of the vertices; the initial conditions; and the order of the sides), the orbit

\footnotetext{
21 Gale was especially proud that Martin Gardner featured his work in "Mathematical Games" columns. Gardner discussed "Bridg-It" in a column that appeared in October 1958 and “Chomp” in January 1973.

22 As the exhibit points out, the Hadwiger and Glur theorem does not generalize to three dimensions. The celebrated impossibility theorem of Max Dehn (see Aigner and Ziegler, 2004, pp. 47-48, for a beautiful proof) solved Hilbert's third problem.
} 
appears. The visitor quickly sees the remarkable fact that for a given ordering, all paths quickly converge to a limiting cycle that is independent of the initial conditions. ${ }^{23}$

The fourth exhibit illustrates the stable marriage problem.

The David Gale Fund for Interactive Mathematics will maintain and enrich MathSite.

\section{Conclusion}

To David Gale, an argument was mathematical if it was "carried out with sufficient precision." He found places for mathematical arguments everywhere and has left us with a rich set of examples. In looking at his work, it is hard to draw a distinction between the "pure" and the "applied." Concrete problems motivate David Gale's work. His papers are accessible because their motivations are familiar and raise tangible questions. Some of Gale's work has had important implications for "real-world" problems. In this sense, the research in applied. On the other hand, his formulations are elegant and abstract. His work has an intrinsic beauty that does not depend on any specific application.

We will always have a record of David Gale's research contributions. These results will never lose their ability to stimulate, to enlighten, and to entertain. His name will always be associated with remarkable ideas. Another legacy is less durable. I made no attempt to convey the extent to which Gale's curiosity and insight extended to a wide range of non-professional interests and how he brought his passion for mathematics to every aspect of his life. Each day he saw new things and the way that his eyes twinkled with delight when he described new problems was infectious. The professional community will remember David Gale for his theorems. Those fortunate enough to know David will preserve still richer memories.

\section{References}

Abdulkadiroglu, Atila, Sönmez, Tayfun, 1999. House allocation with existing tenants. J. Econ. Theory 88 (2), 233-260.

Aigner, Martin, Ziegler, Günter, 2004. Proofs from THE BOOK. Springer-Verlag, Berlin, Heidelberg, New York.

Alkan, Ahmet, Demange, Gabrielle, Gale, David, 1991. Fair allocation of indivisible goods and criteria of justice. Econometrica 59 (4), 1023-1039.

Arrow, Kenneth J., Hahn, Frank H., 1971. General Competitive Analysis. Holden-Day, San Francisco.

Carathéodory, Constantin, 1907. Über den Variabilitätsbereich der Koeffizienten von Potenzreihen, die gegebene Werte nicht annehmen. Math. Ann. 64, 95-115.

Carathéodory, Constantin, 1911. Über den Variabilitätsbereich der Fourierschen Konstanten von positiven harmonischen Funktionen. Rend. Circ. Mat. Palermo 32, 193-217.

Chen, Xi, Deng, Xiaotie, 2006. Settling the complexity of two-player Nash equilibrium. In: FOCS'06: 47th Annual IEEE Symposium on Foundations of Computer Science, pp. 261-272.

Crawford, Vincent P., Knoer, Elsie, 1982. Job matching with heterogeneous firms and workers. Econometrica 49 (2), $437-451$.

Daskalakis, Constantinos, Goldberg, Paul W., Papadimitriou, Christos H., 2006. The complexity of computing a Nash equilibrium. In: STOC '06: Proceedings of the Thirty-Eighth Annual ACM Symposium on Theory of Computing. ACM, New York, NY, USA, pp. 71-78.

Daskalakis, Constantinos, Papadimitriou, Christos H., 2005. Three-player games are hard. Electron. Colloq. Comput. Complex. 139.

Debreu, Gérard, 1956. Market equilibrium. Proc. Natl. Acad. Sci. 42, 876-878.

DeGroot, Morris H., 1974. Reaching a consensus. J. Amer. Statistical Assoc. 69 (345), 118-121.

Demange, Gabrielle, Gale, David, 1985. The strategy structure of two-sided matching markets. Econometrica 53 (4), 873-888.

Demange, Gabrielle, Gale, David, Sotomayor, Marilda, 1986. Multi-item auctions. J. Polit. Economy 94 (4), 863-872.

Dubins, Lester E., Freedman, David A., 1981. Machiavelli and the Gale-Shapley Algorithm. Amer. Math. Monthly 88 (7), 485-494.

Eisenberg, Edmund, Gale, David, 1959. Consensus of subjective probabilities: The pari-mutuel method. Ann. of Math. Statist. 30, 165-168.

Eisenbud, David, Popescu, Sorin, 2000. The projective geometry of the Gale transform. J. Algebra 230 (1), 127-173.

Friedman, Harvey, 1971. Higher set theory and mathematical practice. Ann. of Math. Logic 2, 325-357.

Gale, David, 1955. The law of supply and demand. Math. Scand. 3 (33-44).

Gale, David, 1956a. A closed linear model of production. In: Kuhn, Harold W., Tucker, Albert W. (Eds.), Linear Inequalities and Related Systems. In: Ann. of Math. Stud., vol. 38. Princeton University Press, pp. 285-303 (Chapter 18).

Gale, David, 1956b. Neighboring vertices on a convex polyhedron. In: Kuhn, Harold W., Tucker, Albert W. (Eds.), Linear Inequalities and Related Systems. In: Ann. of Math. Stud., vol. 38. Princeton University Press, pp. 255-263 (Chapter 15).

Gale, David, 1957. A theorem on flows in networks. Pacific J. Math. 7, 1073-1082.

Gale, David, 1960. The Theory of Linear Economic Models. McGraw-Hill.

Gale, David, 1963a. Neighborly and cyclic polytopes. In: Klee, Victor L. (Ed.), Convexity. In: Proc. Sympos. Pure Math., vol. 7. American Mathematical Society, Providence, RI.

Gale, David, 1963b. A note on global instability of competitive equilibrium. Naval Res. Logist. Quart. 10, 81-87.

Gale, David, 1967. On optimal development in a multi-sector economy. Rev. Econ. Stud. 34, 1-18.

Gale, David, 1973. Pure exchange equilibrium of dynamic economic models. J. Econ. Theory 6, 12-26.

Gale, David, 1974. A curious Nim-type game. Amer. Math. Monthly 81, 876-879.

Gale, David, 1998. Tracking the Automatic Ant. Springer-Verlag, New York.

Gale, David, 2001. The two-sided matching problem, origin, development and current issues. Int. Game Theory Rev. 3 (2-3), 237-252.

Gale, David, 2009. Topological games at Princeton, a mathematical memoir. Games Econ. Behav., doi:10.1016/j.geb.2009.04.019.

Gale, David, Kuhn, Harold W., Tucker, Albert W., 1951. Linear programming and the theory of games. In: Koopmans, Tjallings C. (Ed.), Activity Analysis of Production and Allocation. Wiley, New York, pp. 287-297 (Chapter 19).

Gale, David, Mas-Colell, Andreu, 1975. An equilibrium existence theorem for a general model without ordered preferences. J. Math. Econ. 2, 9-15.

Gale, David, Nikaidô, Hukune, 1965. The jacobian matrix and global univalence of mappings. Math. Ann. 2, 81-93.

Gale, David, Shapley, Lloyd S., 1962. College admissions and the stability of marriage. Amer. Math. Monthly 69, 9-15.

Gale, David, Sobel, Joel, 1982. On optimal distribution of output from a jointly owned resource. J. Math. Econ. 9 (1), 51-59.

${ }^{23}$ I leave the elementary proof as an exercise for the reader. 
Gale, David, Sotomayor, Marilda, 1985. Some remarks on the stable matching problem. Discrete Appl. Math. 11 (223-232), 168.

Gale, David, Stewart, Frank M., 1953. Infinite games with perfect information. In: Kuhn, Harold W., Tucker, Albert W. (Eds.), Contributions to the Theory of Games II. In: Ann. of Math. Stud., vol. 28. Princeton University Press, pp. 245-266.

Grünbaum, Branko, 2003. Convex Polytopes, second ed. Springer-Verlag, New York.

Hadwiger, Hugo, Glur, Paul, 1951. Zerlegungsgleichheit ebener Polygone. Elem. Math. 6, 97-106.

Hall, Philip, 1935. On representatives of subsets. J. London Math. Soc. 10, 26-30.

Hatfield, John W., Milgrom, Paul R., 2005. Matching with contracts. Amer. Econ. Rev. 94 (4), 913-935.

Jain, Kamal, Vazirani, Vijay V., 2007. Eisenberg-Gale markets: Algorithms and structural properties. In: STOC '07: Proceedings of the Thirty-Ninth Annual ACM Symposium on Theory of Computing, San Diego, pp. 364-373.

Knuth, Donald E., 1976. Mariages stables et leurs relations avec d'autres problèmes combinatoires: Introduction a l'analyse mathématique des algorithmes. Les Presses de l'Université de Montréal, Montréal.

Knuth, Donald E., 1997. Stable Marriage and Its Relation to Other Combinatorial Problems: An Introduction to the Mathematical Analysis of Algorithms. Crm Proc. Lecture Notes, vol. 10. American Mathematical Society.

Kolata, Gina, 2007a. The myth, the math, the sex. The New York Times 156 (54,034), August 12.

Kolata, Gina, 2007b. The median, the math and the sex. The New York Times 156 (54,041), August 19.

Kuhn, Harold W., 1955. On certain convex polyhedra. Bull. Amer. Math. Soc. 61, 557-558.

Lemke, Carlton E., Howson Jr, Joseph T., 1964. Equilibrium points of bimatrix games. J. Soc. Industr. Appl. Math. 12 (2), 413-423.

Majumdar, Mukul (Ed.), 1992. Equilibrium and Dynamics: Essays in Honour of David Gale. MacMillan Press, London.

Martin, Donald A., 1975. Borel determinacy. Ann. of Math. (2) 102 (2), 363-371.

Motzkin, Theodore S., 1957. Comonotone curves and polyhedra. Bull. Amer. Math. Soc. 63, 35.

Mycielski, Jan, 1964. On the axiom of determinateness. Fund. Math. 53, 205-224.

von Neumann, John, 1937. Über ein ökonomisches Gleichungssystem und eine Verallgemeinerung des Brouwerschen Fixpunktzatzes. Ergeb. Math. Kolloquiums Wien 8.

von Neumann, John, 1945-1946. A model of general economic equilibrium. Rev. Econ. Stud. 13 (1), 1-9.

Nikaidô, Hukukane, 1956. On the classical multilateral exchange problem. Metroeconomica 8, 135-145.

Roth, Alvin E., 1982. The economics of matching: Stability and incentives. Math. Oper. Res. 7, 617-628.

Roth, Alvin E., 2008. Deferred acceptance algorithms: History, theory, practice, and open questions. Int. J. Game Theory 36 (3-4), 537-569.

Roth, Alvin E., Sönmez, Tayfun, Utku Ünver, M., 2004. Kidney exchange. Quart. J. Econ. 119 (2), 457-488.

Roth, Alvin E., Sotomayor, Marilda, 1990. Two-Sided Matching: A Study in Game-Theoretic Modeling and Analysis. Econometric Soc. Monogr. Ser. Cambridge University Press, Cambridge.

Samuelson, Paul A., 1958. An exact consumption-loan model of interest with or without the social contrivance of money. J. Polit. Economy 66 (6), 467-482. Savani, Rahul, von Stengel, Bernhard, 2006. Hard-to-solve bimatrix games. Econometrica 74 (2), 397-429.

Shapley, Lloyd S., Scarf, Herbert E., 1974. On cores and indivisibility. J. Math. Econ. 1, 23-37.

Shapley, Lloyd S., Shubik, Martin, 1971. The assignment game I: The core. Int. J. Game Theory 1 (1), 111-130.

Wolfe, Philip, 1955. The strict determinateness of certain infinite games. Pacific J. Math. 5 (Suppl. 1), 841-847. 